\title{
Echtzeit-Rekonstruktion von Magnetresonanz-Bildern
}

Crelier, Gérard R ; Fischer, Stefan E ; Arm, Erich ; Kunz, Patrik ; Boesiger, Peter

DOI: https://doi.org/10.1515/bmte.1993.38.s1.361

Posted at the Zurich Open Repository and Archive, University of Zurich ZORA URL: https://doi.org/10.5167/uzh-155344

Journal Article

Published Version

Originally published at:

Crelier, Gérard R; Fischer, Stefan E; Arm, Erich; Kunz, Patrik; Boesiger, Peter (1993). EchtzeitRekonstruktion von Magnetresonanz-Bildern. Biomedizinische Technik. Biomedical engineering, 38(s1):361362.

DOI: https://doi.org/10.1515/bmte.1993.38.s1.361 


\title{
Echtzeit-Rekonstruktion von Magnetresonanz-Bildern
}

\author{
Gérarll R. Crelier, Stefan E. Fischer, Erich Arm, Patrik Kunz, Peter Boesiger
}

Institut für Biomedizinische Technik und Mcdizinische Informatik der Universităt Zürich und der Eidgenössischen Technischen Hochschule Zürich, Schweiz

\section{Einleitung}

Neue Verfahren in der bildgebenden Magnetresonanz (MRI) crlauben die Aufnahme eines Bildes in weniger als $100 \mathrm{~ms}$. Damit ist es prinzipiell möglich, operative Eingriffe an Patienten unter Kontrolle von MRI durchzufüren (interventionelle Magnetresonanz-Bildgebung). Neben vielen anderen Problemen, die für derartige Techniken zu lösen sind, besteht auch dasjenige der schnellen Rekonstruktion der MR-Bilder. Diese besteht im wesentlichen aus einer zeitaufwendigen, zweidimensionalen komplexen Fourier-Transformation der gemessenen Echodaten. Gängige Rekonstruktionssysteme benötigen dazu typischerweise 1 bis 2 Sekunden. Für interventionelle Magnetresonanz besteht demnach ein klares Bedürfnis für ein Echtzeit-Rekonstruktionssystem. Im Weiteren sind Messverfahren in Entwicklung, bei denen sich schon nach wenigen gemessenen MR-Echos beispielsweise der Bildkontrast beurteilen lässt, worauf dieser noch während der Messung durch Änderung von Messparametern verbessert werden könnte. Auch solche interaktive Messverfahren sind auf eine Echtzeit-Rekonstruktion angewiesen. In diesem Beitrag wird ein neu entwickeltes, flexibles System zur Echtzeit-Rekonstruktion von Magnetresonanz-Bildern, die mit dem konventionellen Fourier-Abbildungsverfahren aufgenommen werden, vorgestellt.

\section{Methode}

Das System wurde so konzipiert, dass es der Vielfalt von Messverfahren eines MR-Tomographen gerecht wird und Bilder mit beliebigen Aufnahmeprotokollen rekonstruieren kann.

Die Figur 1 zeigt das Blockschaltbild der implementierten Hardware. Bei MR-Tomographen fallen die Echodaten, welche aus bis zu 1024 komplexen Abtastwerten bestehen können, mit der Repetitonszeit TR an. Die maximale Abtastfrequenz beträgt dabei $128 \mathrm{kHz}$ bei einer Auflösung von 16 Bit. Die digitalen Echodaten werden über einen optischen, seriellen Link (5MBaud) der Rekonstruktionshardware zugeführt.
Ein Signalprozessor (TMS320C31 DSP, Texas Instruments) holt die Daten am Eingangsinterface ab und führt vorerst eine Skalienung, Offsetunterdrükkung und Phasenkorrektur aus. Anschliessend wird eine eindimensionale, komplexe Fourier-Transformation ausgeführt. Die Länge der verwendeten FFT wird dabei der Echolänge angepasst. Das ebenfalls komplexe Resultat der FFT wird auf 256 Datenpunkte reduziert oder interpoliert und in einem Speicher als Zeile eines Hybrid-K-Raumes (x, ky) abgelegt. Ein

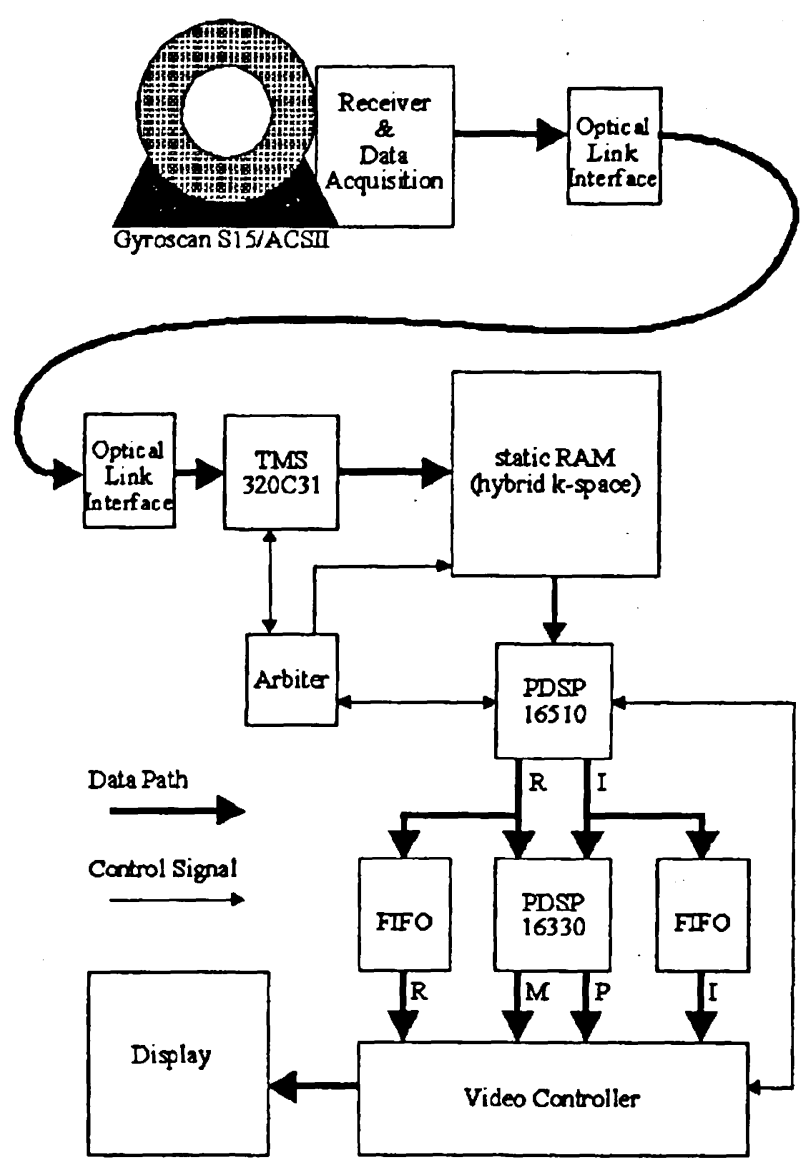

Figur 1: Blockschaltbild Rekonstruktionssystem 
spezieller FFT-Prozessor (PDSP16510, GEC Plessey) führt unabhängig davon und kontinuierlich eine eindimensionale Fourier-Transformation der 256 komplexen Kolonnen des Hybrid-K-Raum Speichers durch. Die Ausgangsdaten des FFT-Prozessors (Realund Imaginärteil) werden mittels eines Pythagorasprozessors (PDSP16330, GEC Plessey) in Betrag und Phase transformiert. Ein intelligenter Videospeicher mit einem Signalprozessor (TMS320C25, Texas Instruments) stellt das nun rekonstruierte Bild dar, wobei zwischen Amplituden- (M), Phasen- (P), Real- (R) oder Imaginärbild (I) ausgewählt werden kann. Dabei wird die Arbeitsgeschwindigkeit des FFT-Prozessors mit der Bildwiederholfrequenz synchronisiert.

Das Rekonstruktions-System wurde als Einsteckkarte für einen Personal Computer realisiert und an einen MR-Tomographen (Philips Gyroscan S15/ACS II) angeschlossen.

\section{Ergebnisse}

Das Rekonstruktions-System läuft parallel zum bestehenden Rekonstruktionspfad des Tomographen ohne diesen in irgend einer Weise zu beeinflussen. Bilder bis zur Standardauflösung von 256x256 Pixel werden rekonstruiert. Das System lässt sich programmieren, um jedes vom Tomographen unterstützte Aufnahmeprotokoll zu verarbeiten. Die Datenverarbeitung in Ausleserichtung der Echos wird vollständig vom programmierbaren Signalprozessor TMS320C31 erledigt. Seine Leistungsfähigkeit von 16.7 MIPS erlaubt es zum Beispiel, eine komplexe 512-Punkt FFT in 1.79 ms durchzuführen. Mit der benötigten Zeit für Datentransfer, Offsetunterdrückung, Phasenkorrektur und Fourier-Transformation ergeben sich die in Tabelle 1 aufgeführten minimalen Repetitionszeiten TR furr entsprechende Echolängen.

\begin{tabular}{|c|c|c|}
\hline Echolänge & FFT & $\min . \mathrm{TR}$ \\
\hline 1024 & $2.37 \mathrm{~ms}$ & $8.2 \mathrm{~ms}$ \\
\hline 512 & $1.79 \mathrm{~ms}$ & $4.0 \mathrm{~ms}$ \\
\hline 256 & $0.62 \mathrm{~ms}$ & $1.8 \mathrm{~ms}$ \\
\hline 128 & $0.37 \mathrm{~ms}$ & $0.8 \mathrm{~ms}$ \\
\hline
\end{tabular}

Tabelle 1: Minimale Repetitionszeiten TR
Die Fourier-Transformation in Phasenkodierrichtung wird vom FFT-Chip durchgeführt. Eine Kolonne aus dem Hybrid-K-Raum von 256 komplexen Werten wird dabei in $53 \mu$ s transformiert. Damit ist der ganze Hybrid-K-Raum in $13.6 \mathrm{~ms}$ verarbeitet und es lassen sich Bildwiederholraten von bis zu $70 \mathrm{~Hz}$ erzielen. Der verwendete intelligente Bildspeicher erlaubt jedoch lediglich eine Bildwiederholrate von $30 \mathrm{~Hz}$. Dafür sind einfache Bildverarbeitungsroutinen wie Zooming und Bildkontrastbeeinflussung implementiert und könnén durch weitere ergänzt werden.

Bis maximal 4 verschiedene Schichtbilder (zum Beispiel aus einer Multislice-Messung) lassen sich gleichzeitig rekonstruieren und je als Amplituden-, Phasen-, Real- und Imaginärbild darstellen. Das System erlaubt auch Bilder zu rekonstruieren und darzustellen, von denen noch nicht die gesamte Information vorliegt (etwa während langsamen Spinecho-Sequenzen). Bei entsprechenden Messprotokollen lassen sich somit schon frühzeitig Bildkontrast und Schichtposition aus den niederfrequenten Anteilen des Hybrid-K-Raumes erkennen und beurteilen. In Echtzeit lässt sich dann verfolgen, wie hochfrequente Anteile zur Verbesserung der Bildschärfe beitragen.

\section{Diskussion}

Dank der Verwendung von modernen, spezialisierten Bausteinen ist ein Echtzeit-Rekonstruktionssystem von MR-Bildern möglich geworden. Zum Aufbau entsprechender Hardware betragen die Komponentenkosten weniger als 2000 US\$. Durch die Flexibilität des Signalprozessors am Eingang des Systems können Aufnahmen mit beliebigen Messprotokollen durchgeführt werden. Wenn in Zukunft ultraschnelle Messverfahren weiter verbessert werden können und noch schnellere Repetitionszeiten TR erlauben, so kann die benötigte Verarbeitungszeit der Echos in Ausleserichtung durch den Signalprozessor limitierend wirken. Die Aufgaben, die der Signalprozessor im gegenwärtigen System wahmimmt, liessen sich dazu in einer erweiterten Version relativ leicht auf mehrere, parallel arbeitende Prozessoren aufteilen. Dadurch würden die minimal möglichen Repetitionszeiten TR um die Anzahl Prozessoren dividiert.

Die Verarbeitung in Phasenkodicrrichtung erfolgt bereits jetzt in Echtzcit und dic maximale Bildwiederholfrequenz von $70 \mathrm{~Hz}$ crfullt die Anfordenungen der interventioncllen MR-Verfahren sowie die der zeitsparenden, interaktiven Messstrategien. 